\title{
Author Correction: Mammal diversity influences the carbon cycle through trophic interactions in the Amazon
}

Mar Sobral, Kirsten M. Silvius, Han Overman, Luiz F. B. Oliveira, Ted K. Raab and José M. V. Fragoso

Correction to: Nature Ecology \& Evolution https://doi.org/10.1038/s41559-017-0334-0 (2017); published online 9 October 2017.

In the version of this Article originally published, the surname of Ted K. Raab was misspelt. This error has now been corrected in all versions of the Article.

Published online: 19 October 2017

https://doi.org/10.1038/s41559-017-0376-3 